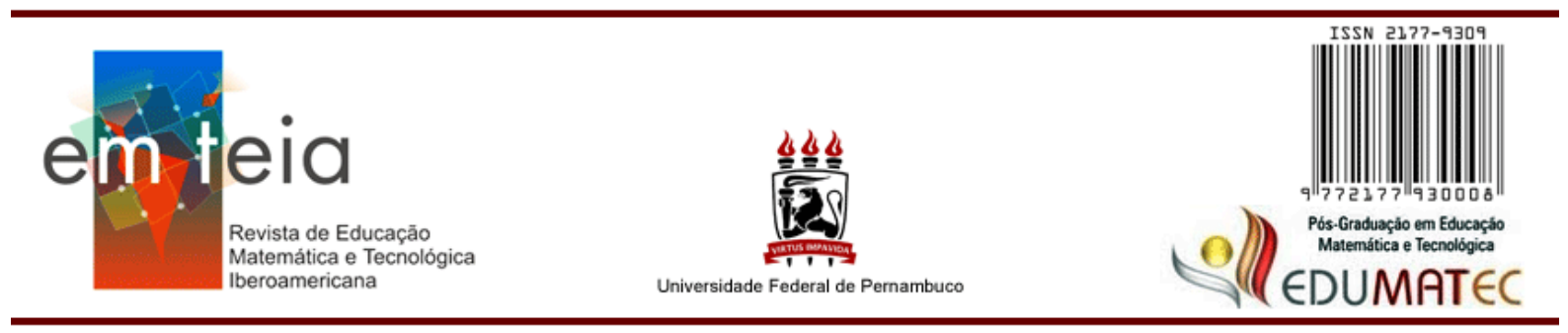

O GEOGEBRA COMO FERRAMENTA AO ENSINO DAS FUNÇÕES TRIGONOMÉTRICAS NO ENSINO MÉDIO: Um estudo de caso no IFMT Campus Cuiabá GEOGEBRA AS A TOOL FOR TEACHING TRIGONOMETRIC FUNCTIONS IN HIGH SCHOOL: A case study at IFMT Campus Cuiabá

\author{
Carlos Carlão Pereira do Nascimento \\ Mestre em Ensino de Ciências Exatas \\ Professor do IFMT Campus Cuiabá. \\ IFMT - Mato Grosso - Brasil \\ carlaopnascimento@gmail.com \\ Maria Madalena Dullius \\ Doutora em Ensino de Ciências e Matemática \\ Professora da Universidade do Vale do Taquari. Univates - Rio Grande do Sul - Brasil \\ madalena@univates.br \\ André Krindges \\ Doutor em Matemática Aplicada \\ Professor da Universidade Federal de Cuiabá - UFMT. \\ UFMT - Mato Grosso - Brasil \\ krindges@gmail.com
}

\title{
Resumo
}

O presente artigo é um recorte da dissertação de mestrado intitulada "O uso do GeoGebra no Ensino das Funções Trigonométricas no $2^{\circ}$ ano do Ensino Médio no IFMT Campus Cuiabá" (NASCIMENTO, 2019a) que investigou como a utilização do software GeoGebra pode potencializar a exploração de tópicos da trigonometria. O objetivo principal foi investigar como a utilização do software GeoGebra pode potencializar a exploração das Funções Trigonométricas seno, cosseno e tangente. De cunho qualitativo, a pesquisa foi realizada no Instituto Federal de Educação, Ciência e Tecnologia de Mato Grosso - IFMT Campus Cuiabá com estudantes do segundo ano do Ensino Médio Integrado ao Curso Técnico de Informática. Para o desenvolvimento propôs-se uma intervenção pedagógica realizada por meio do software GeoGebra a partir de uma sequência didática. Neste trabalho apresentamos algumas atividades desenvolvidas durante o estudo bem como a análise das mesmas. Como referencial teórico nos pautamos em Borba (2016), Dullius e Quartieri (2015), Gravina et al. (2012), Valente (1997), entre outros.

Palavras-Chave: software GeoGebra. Funções trigonométricas. Sequência didática. Ensino médio. Intervenção pedagógica. 


\begin{abstract}
This paper is part of a master's thesis titled "The Use of GeoGebra at the Teaching of Trigonometric Functions in the 2nd School Year of High School at IFMT Campus Cuiabá" (NASCIMENTO, 2019a). The research investigated what way the use of the GeoGebra software may improve the study of trigonometry topics, specifically the exploration of the sine, cosine and tangent trigonometric functions. The study counted on two main objectives: a) developing a didactic sequence using the GeoGebra software for studying trigonometric functions; b) to promote student involvement in studying trigonometry by using the software. The study is characterized as qualitative type and the research was carried out at the Federal Institute of Education, Science and Technology of Mato Grosso - IFMT Campus Cuiabá. The participants were second year students from the High School Integrated to the Technical Course of Computing. It was proposed as a pedagogical intervention using the GeoGebra software, accordingly a didactic sequence systematized in twenty-four classes. The research was guided on the theoretical framework proposed by Borba (2016), Dullius and Quartieri (2015), Gravina et al. (2012), Valente (1997), among other researchers.
\end{abstract}

Keywords: software GeoGebra. Trigonometric functions. Didactic sequence for teaching Trigonometry. High school. Pedagogical intervention.

\title{
Introdução
}

Ensinar não se configura como uma tarefa simples ou fácil; em se tratando do ensino de Matemática, mais especificamente, ao ensino da Trigonometria, o desafio é ainda maior. Na perspectiva de dinamizar e potencializar a forma de ensinar Trigonometria, investigamos seu ensino por meio do uso do software GeoGebra.

A pesquisa foi desenvolvida no âmbito do mestrado profissional em Ciências Exatas da Universidade do Vale do Taquari Univates, na linha de pesquisa Tecnologias, Metodologias e Recursos Didáticos para o Ensino de Ciência e Matemática. O objetivo foi investigar como a utilização do software GeoGebra pode potencializar a exploração de tópicos da trigonometria. Para tanto, foi desenvolvida uma sequência didática com estudantes $2^{\circ}$ ano do Ensino Médio do Instituto Federal do Mato Grosso, Campus Cuiabá. (NASCIMENTO, 2019b).

A motivação para realizar esta pesquisa decorreu de uma série de fatores. Na condição de docente, a partir da experiência em sala de aula ministrando a disciplina de Matemática, o primeiro autor desse trabalho observou que os exercícios e provas desenvolvidos pelos discentes demonstravam que eles não compreendiam o conteúdo da disciplina. Da mesma forma, não compreendiam a linguagem simbólica utilizada neste conteúdo, como consequência não conseguiam compreender a Trigonometria como um instrumento matemático com capacidade para resolver problemas em um dado contexto.

Considerando estas constatações, buscaram-se alternativas para o ensino que estivessem relacionadas ao uso de tecnologias por perceber a dinâmica da sociedade e da 
importância do professor e escola utilizarem cada vez mais recursos atuais para tornar o processo de construção do conhecimento um exercício prazeroso e interessante.

Para atender ao objetivo proposto para esta investigação, a opção metodológica foi pela pesquisa qualitativa, realizada no Instituto Federal de Educação, Ciência e Tecnologia de Mato Grosso - IFMT Campus Cuiabá com estudantes do $2^{\circ}$ ano do Ensino Médio Integrado ao Curso Técnico em Informática. Para o desenvolvimento da pesquisa foi proposta uma intervenção pedagógica realizada por meio do software GeoGebra a partir de uma sequência didática sistematizada em vinte e quatro aulas. Para análise dos dados utilizou-se metodologia descritiva. Neste artigo serão apresentadas somente algumas das atividades desenvolvidas, bem como a análise das mesmas. No que tange ao uso de recursos digitais para as aulas, usouse como base o referencial teórico de Borba (2016), Dullius e Quartieri (2015), Gravina et al. (2012), Valente (1997), entre outros.

\section{Tecnologias digitais no ensino da Matemática}

Neide e Quartieri (2016) apontam vários fatores que podem ser apresentados como justificativas para a utilização de tecnologias nas salas de aula, como o uso de computadores, tablets e celulares. Segundo os autores, a preocupação não é mais o porquê utilizar, uma vez que há a necessidade de utilização dessas tecnologias, mas de como utilizar esses recursos tecnológicos nos processos de ensino (NEIDE; QUARTIERI, 2016).

Nessa conjuntura, torna-se indispensável a familiarização do professor em utilizar os recursos tecnológicos junto aos estudantes para além da metodologia tradicional, viabilizando dessa forma, importantes contribuições, tais como,

\footnotetext{
Apresentar, de diferentes formas, um mesmo elemento do conteúdo programático pode ajudar o aluno a compreender o tema que estás sendo estudado. Além de revisitar, explorar o assunto via imagens ou animações, privilegiam o fazer pedagógico em sala de aula. A visualização é uma ação importante para a construção da aprendizagem, principalmente na área das Ciências Exatas (NEIDE; QUARTIERI, 2016, p. 10).
}

Com previsão no art. 35-A da Constituição Federal, o documento nomeado Base Nacional Comum Curricular - BNCC (BRASIL, 2017) define direitos e objetivos de aprendizagem no Ensino Médio, em consonância com as diretrizes do Conselho Nacional de Educação em diferentes áreas do conhecimento, dentre essas áreas destaca-se a Matemática e suas tecnologias e evidencia a importância de assegurar a aprendizagem e o desenvolvimento 
do estudante em utilizar conceitos, procedimentos e estratégias de forma que possibilite ir além de resolver os problemas:

No Ensino Médio, na área de Matemática e suas Tecnologias, os estudantes devem utilizar conceitos, procedimentos e estratégias não apenas para resolver problemas, mas também para formulá-los, descrever dados, selecionar modelos matemáticos e desenvolver o pensamento computacional, por meio da utilização de diferentes recursos da área (BRASIL, 2017, p. 470, grifo nosso).

Assim, o desenvolvimento do pensamento computacional torna-se um imperativo na prática docente. Nesse entendimento, em se tratando do uso das tecnologias digitais, Paiva (2016, p. 22-23) ressalta:

\footnotetext{
É fundamental reconhecer que as tecnologias adentraram na vida humana num ritmo e sem volta. Sendo assim, faz-se necessário assimilá-las como parte de um processo natural de evolução da cultura da sociedade. Esta ideia ganha força ao lembrar a origem e o desenvolvimento da espécie humana e perceber a importância das mudanças para nossa evolução, e que foram, e até hoje são, essenciais para nossa existência e adaptabilidade ao meio. [...] No campo educacional, um desses atores são os professores, e são deles, ou melhor, de parte deles, uma das frentes de resistência em aceitar o uso das tecnologias como ambiente facilitador para o processo de ensino e de aprendizagem.
}

O contexto educativo não está imune aos benefícios da tecnologia. $\mathrm{Na}$ atualidade, seja para ensinar ou aprender, os recursos tecnológicos se constituem meios importantes para potencializar as práticas educativas. Fica perceptível que, mesmo diante da importância e espaço que as tecnologias digitais ocupam no campo educativo, ao professor recai a responsabilidade quanto ao uso profícuo desta tecnologia. Assim, no que tange ao ensino de Matemática, os softwares se constituem em possibilidades viáveis de articulação entre tecnologia digital, ensino, aprendizagem e desenvolvimento cognitivo (AMADO; CARREIRA, 2015).

Diante do exposto, o desafio docente maior consiste em ensinar na era da tecnologia. Conforme menciona Borba (2016, p. 56), “[...] As inovações educacionais, em sua grande maioria, pressupõem mudança na prática docente, não sendo uma exigência exclusiva daquelas que envolvem o uso da tecnologia em informática". O autor afirma ainda que "À medida que a tecnologia informática se desenvolve, nos deparamos com a necessidade de atualização de nossos conhecimentos sobre o conteúdo ao qual ela está sendo integrada" (BORBA, 2016, p. 64). É nesse sentido que compreendemos a importância do software GeoGebra no ensino de trigonometria e ao mesmo tempo da dinamização da forma de ensinar.

O uso inteligente do computador está vinculado à maneira como a tarefa será realizada por ele, ou seja, relacionada a uma prática dinâmica, e não somente um atributo inerente ao 
mesmo, como se fosse garantia de uma aula diferente do que seria uma aula tradicional, de quadro de giz (VALENTE, 1997).

Nesse mesmo entendimento, Dullius e Quartieri (2015) ressaltam que nem sempre o uso do computador está relacionado a uma prática dinâmica, uma vez que a utilização de tecnologias está sujeita a uma variedade de condicionantes. Assim, mesmo que os professores utilizem o computador em sala de aula, em alguns casos, esse uso ocorre de forma tradicional:

\begin{abstract}
Havendo casos em que a tecnologia não passa de um acessório numa prática pedagógica tradicional. As mudanças proporcionadas por esses recursos representam um desafio a ser incorporado no cotidiano da escola, levando em conta que a prática docente pouco mudou ao longo do tempo, diferentemente dos alunos (DULLIUS; QUARTIERI, 2015, p. 5).
\end{abstract}

Esse uso tradicionalista torna restritos os resultados positivos decorrentes da utilização de computadores, pois este uso é direcionado apenas para o campo teórico e acadêmico, como apontam os autores:

Este potencial (uso do computador) ainda não tem sido devidamente explorado e integrado ao cotidiano da prática escolar, ficando restrito a discussões teóricas e acadêmicas. Para as escolas e para muitos professores, as tecnologias continuam a ser um corpo estranho, que provoca sobretudo incomodidade. O receio de ficar pra trás tem levado a escola a investir na compra de equipamentos, muitas vezes deixando para segundo plano o ensino das novas tecnologias (DULLIUS; HAETINGER, 2004, p. 3).

Uma estratégia de superação dessas limitações seria a posse, pelo professor, de um bom aplicativo computacional, o qual seria utilizado conforme a disposição da turma: A facilidade com que esses podem explorar e verificar o que acontece com várias
situações análogas é útil para formar ou testar suas convicções, levando-os a
formular conjecturas, aguçando sua curiosidade para buscar uma demonstração.
Bom aplicativos computacionais, devidamente utilizados, permitem testar a
capacidade de transferência de conhecimentos dos estudantes, a potencialidade de
sua mobilidade em vários contextos e a adaptabilidade dos instrumentos (DULLIUS;
HAETINGER, 2004, p. 4).

Outros desafios ainda necessitam serem transpostos, como a precarização do trabalho docente bem como as limitações das condições físicas e materiais das escolas públicas. Nesse aspecto, apesar do Plano Nacional de Educação - PNE (BRASIL, 2014-2024) definir um aumento do financiamento da educação, previsto na meta 20 , constitui uma adversidade dada à deficiência recorrente na história da educação brasileira. Assim, para que se obtenha uma referência mínima de qualidade a nível de ensino e infraestrutura é preciso que ocorra, de fato, 
o incentivo de políticas de valorização docente, como uma formação sólida e salários dignos, por exemplo.

Ao desenvolver uma atividade de ensino com discentes do Ensino Médio por meio do conteúdo de trigonometria utilizando as possibilidades do software GeoGebra, os autores Lima, Carvalho e Morgado (2005) destacam que entre os potenciais oferecidos pelo software estão a construção, o dinamismo, a investigação, a visualização e a argumentação. Nesse mesmo entendimento, Scaldelai (2014) ressalta que, "Em se tratando de um software dinâmico, gráficos, álgebra e tabelas são conectados dinamicamente, ou seja, cada elemento que é alterado na janela de álgebra, também sofre alteração na janela gráfica e na de cálculo e vice-versa" (SCALDELAI, 2014, p. 16-17).

Assim, a construção das figuras pode ser um recurso didático na construção das argumentações dedutivas feitas pelos estudantes, uma habilidade, que segundo Gravina et al. (2012, p. 42), “não deveria ser negligenciada na formação matemática escolar”. O software possui inúmeras ferramentas e comandos possíveis para serem utilizados em atividades pedagógicas. Uma parte dessa gama de possibilidades foi desenvolvida por meio da sequência didática elaborada pelo pesquisador e proposta a uma turma de discentes do Ensino Médio.

\section{Procedimentos metodológicos}

O presente trabalho é um estudo qualitativo, envolvendo um grupo de estudantes do Ensino Médio Integrado que desenvolveram uma sequência didática mediante uso de um recurso computacional, o software GeoGebra, tendo a Trigonometria como elemento fundante desta experiência. A propriedade da pesquisa qualitativa foi ao encontro dos objetivos da pesquisa desenvolvida, uma vez que este permitiu maior acuidade na averiguação das atividades desenvolvidas pelos estudantes participantes.

O cenário da pesquisa foi o Instituto Federal de Mato Grosso - IFMT Campus Cuiabá, localizado no centro do município de Cuiabá. Em termos tecnológicos, o referido Campus conta com uma infraestrutura básica, com diferentes laboratórios para atender aos diversos níveis e modalidades de ensino.

Para a realização da presente pesquisa, foi solicitada a permissão para o diretor geral do Campus. Os sujeitos que fizeram parte dessa pesquisa são de uma turma de trinta e seis estudantes, com idade média de quinze anos, do $2^{\circ}$ ano do Ensino Médio Integrado do Curso de Informática. Trata-se de um curso anual com duração de três anos. A escolha dos sujeitos deu-se em razão de ser uma turma na qual o pesquisador da presente pesquisa ministrava 
aulas de Matemática, sendo o conteúdo de Trigonometria ofertado no $2^{\circ}$ ano do referido curso. Por serem estudantes do Curso de Informática e terem, durante o curso, aulas práticas de Informática no laboratório, já estavam familiarizados com o uso de tecnologias.

Para garantir o anonimato dos participantes, os discentes foram nomeados por “estudantes E1, E2, E3...E36" e assim, sucessivamente, em substituição de seus nomes. A intervenção pedagógica proposta para o desenvolvimento desta pesquisa foi realizada em um laboratório de informática que dispunha de computadores suficientes para que todos os estudantes da turma desenvolvessem, de forma individual, a sequência didática por meio do uso do software GeoGebra, previamente instalado nos computadores. Em cada aula, os estudantes receberam um planejamento das atividades que deveriam ser desenvolvidas. Ao final de cada encontro, os estudantes encaminharam os gráficos construídos para o e-mail do professor e também foram recolhidas as atividades, com o objetivo de identificar as dúvidas mais frequentes, bem como sanar as dificuldades encontradas.

A análise dos dados foi realizada por meio da descrição que consistiu em analisar se os elementos da teoria se articulam aos elementos da prática e em que medida, destacando os condicionantes desta relação. As atividades foram descritas em ordem cronológica, em razão de terem ocorrido em dias diferentes. Nesta perspectiva, Gil (2008, p. 28) aponta: “As pesquisas deste tipo têm como objetivo primordial a descrição das características de determinada população ou fenômeno ou o estabelecimento de relações entre variáveis".

A seguir, apresentamos um recorte da análise do resultado da intervenção pedagógica desenvolvida junto aos estudantes (NASCIMENTO, 2019b). Utilizaram-se as atividades desenvolvidas individualmente por cada estudante, observando se conseguiram correlacionar as atividades propostas com o conteúdo trabalhado de acordo com o objetivo de cada aula. Por meio dos registros das atividades realizadas foi possível detectar os erros e acertos de cada estudante, bem como utilizaram-se registros do caderno de campo do pesquisador para complementação às análises de dados.

\section{Análise dos resultados}

Para a análise dos resultados apresentamos um recorte da pesquisa, destacando algumas atividades desenvolvidas durante a intervenção pedagógica. Em cada aula foram apresentados as questões e os objetivos a serem atingidos seguidos de materiais desenvolvidos dos estudantes e finalizando com a análise. 


\section{Atividades sobre Trigonometria no círculo trigonométrico: Seno, Cosseno e Tangente}

Com estas atividades teve-se como objetivo definir as principais razões trigonométricas no círculo trigonométrico: Seno, Cosseno e Tangente, bem como visualizar a trigonometria no triângulo retângulo. No primeiro momento foram exploradas atividades relacionadas à construção do seno e cosseno. Na segunda parte resultou a construção da tangente. Inicialmente foi sugerida construção do círculo trigonométrico, cujo exemplo de um estudante apresentamos na Figura 6.

Figura 6 - Construção do Círculo Trigonométrico pelo estudante E8

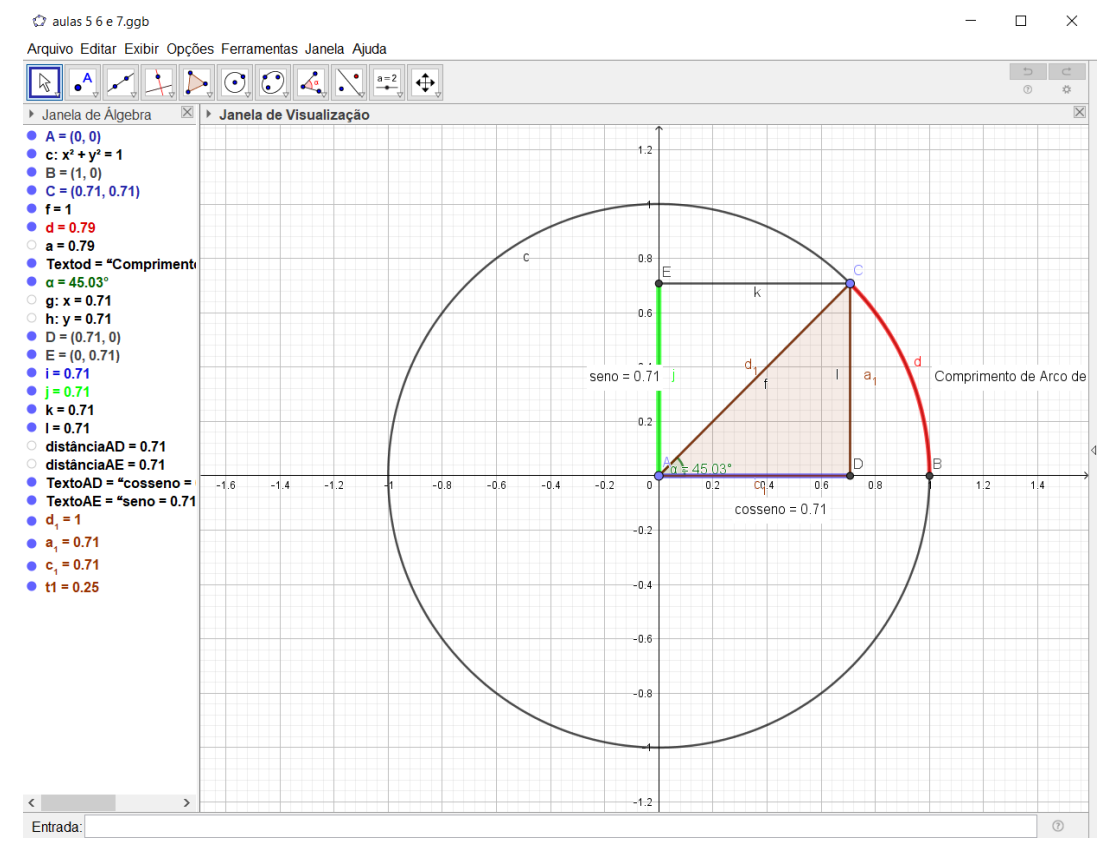

Fonte: Elaboração E8 (2019).

Após gerar o círculo trigonométrico, os estudantes responderam questões referentes ao conteúdo explorado. Dos vinte e nove participantes, vinte acertaram todas as questões e nove erraram alguns dos itens propostos.

Observou-se que, para o desenvolvimento dessa atividade, o uso do software facilitou que os estudantes visualizassem, no triângulo retângulo, o lado que representava o seno e o cosseno, assim como visualizar, no círculo trigonométrico, os sinais em cada quadrante do seno e cosseno e determinar os valores de seno e cosseno, a partir dos ângulos dados em graus e radianos. Na Figura 7, destacamos o exercício resolvido pelo estudante E31: 
Figura 7 - Resolução da atividade 2, segunda parte, estudante E31

Atividade 2

1) Observando a construção feita no GeoGebra, responda os itens: a) Qual segmento representa o seno do arco $B C$ (ou ângulo $B \hat{A} C$ )? $A E$

b) Qual segmento representa o cosseno do arco $\mathrm{BC}$ (ou ângulo $\mathrm{BA} C$ )? $\widehat{\mathrm{AD}}$

2) Movimente o ponto $C$ observando o sinal de seno e cosseno. Com base nisso, preencha a tabela com "positivo" ou "negativo":

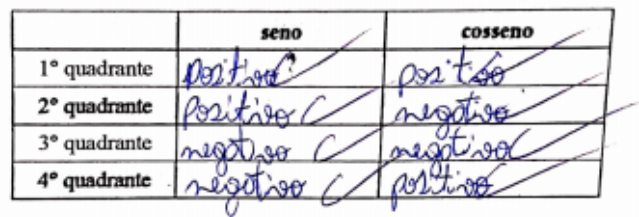

3) Movimente o ponto $\mathrm{C}$ de forma a obter o seno e cosseno dos seguintes ângulos:

\begin{tabular}{|l|l|l|}
\hline & \multicolumn{1}{|c|}{ seno } & cosseno \\
\hline $30^{\circ}$ & 0.5 & 0.87 \\
\hline $45^{\circ}$ & 0.71 & 0.71 \\
\hline $60^{\circ}$ & 0.87 & 0.5 \\
\hline $120^{\circ}$ & 0.87 & -0.5 \\
\hline $135^{\circ}$ & 0.71 & -0.71 \\
\hline $150^{\circ}$ & 0.5 & -0.87 \\
\hline $210^{\circ}$ & -0.5 & -0.87 \\
\hline $225^{\circ}$ & -0.710 & -0.71 \\
\hline $240^{\circ}$ & -0.87 & -0.5 \\
\hline $300^{\circ}$ & -0.87 & 0.5 \\
\hline $315^{\circ}$ & -0.71 & 0.71 \\
\hline $330^{\circ}$ & -0.5 & 0.87 \\
\hline &
\end{tabular}

Fonte: Elaboração E31 (2019).

Com relação aos estudantes que erraram alguns itens da atividade, observou-se que os mesmos construíram os devidos gráficos, entretanto, não souberam extrair os valores dos senos e cossenos de cada ângulo. De acordo com os erros observados dos nove alunos, alguns não conseguiram visualizar na figura o lado do triângulo que representava o seno e o cosseno, nem mesmo identificar e comparar os valores de seno e cosseno de determinados ângulos.

$\mathrm{Na}$ Figura 8 apresentamos material produzido pelo estudante E28 relacionado ao desenvolvimento da tangente. 
Figura 8 - Solução apresentada pelo estudante E28

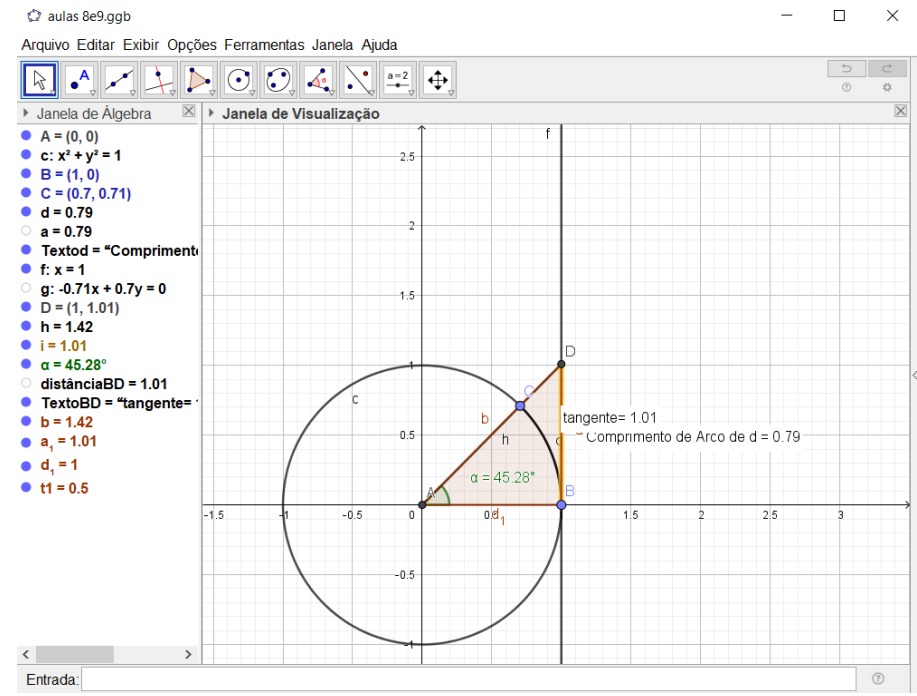

Fonte: Elaboração E28 (2019).

Após gerar o gráfico, o círculo trigonométrico, os estudantes responderam a Atividade 3 (FIGURA 9) que continha seis questões. Dos trinta e um estudantes, vinte e três acertaram todos os itens e oito erraram alguns dos itens propostos. A título de exemplo, destacamos na Figura 9, material produzido pelo estudante E31:

Figura 9 - Resolução da atividade 3, estudante E31

Atividade 3

1) Observando a construção fẹta no GeoGebra, qual segmento representa a tangente do arco $\mathrm{BC}$ (ou ângulo $\mathrm{BÂC}$ )? $\hat{\mathrm{A}}$ )

2) Movimente o ponto $C$ observando o sinal da tangente. Com base nisso, preencha a tabela com "positivo" ou "negativo":

\begin{tabular}{|l|l|}
\hline & \\
\hline $1^{\circ}$ quadrante & posituco \\
\hline $2^{\circ}$ quadrante & megatute \\
\hline $3^{\circ}$ quadrante & positivio \\
\hline $4^{\circ}$ quadrante & negatiot \\
\hline
\end{tabular}

3) Movimente o ponto $\mathrm{C}$ de forma a obter a tangente dos seguintes ângulos:

\begin{tabular}{|c|c|}
\hline & \multicolumn{1}{|c|}{ tangente } \\
\hline $30^{\circ}$ & 0,58 \\
\hline $45^{\circ}$ & 1 \\
\hline $60^{\circ}$ & 1,73 \\
\hline $120^{\circ}$ & $-1,73$ \\
\hline $135^{\circ}$ & -1 \\
\hline $150^{\circ}$ & $-0,59$ \\
\hline $210^{\circ}$ & 0,58 \\
\hline $225^{\circ}$ & 1 \\
\hline $240^{\circ}$ & 1,73 \\
\hline $300^{\circ}$ & $-1,73$ \\
\hline $315^{\circ}$ & -1 \\
\hline $330^{\circ}$ & $-0,58$ \\
\hline
\end{tabular}

Fonte: Elaboração E31 (2019). 
Na Atividade 3, foi possível observar que os estudantes conseguiram visualizar o lado do triângulo que representa a tangente, identificaram os sinais da tangente nos quatro quadrantes, conseguiram visualizar os valores da tangente de vários ângulos, bem como relacionaram a tangente de um ângulo com o seno e o cosseno e compararam o valor da tangente de um ângulo com outros ângulos.

Presumiu-se que o grupo de estudantes que acertou todas as questões conseguiu visualizar no círculo os valores da tangente de vários ângulos, bem como relacionar os valores de seno, cosseno e tangente, tanto no software como no cálculo manual. Esse processo investigativo desencadeia habilidades a serviço de várias competências como utilizar estratégias, conceitos e procedimentos matemáticos, articular conhecimentos, compreender registros, investigar e estabelecer conjecturas que são competências específicas de Matemática e suas tecnologias, de acordo com a Base Nacional Curricular Comum - BNCC (BRASIL, 2017).

A experiência dos estudantes com a manipulação do círculo trigonométrico no software permitiu, ainda, a obtenção de valores de seno, cosseno e tangente sob a forma de números decimais aproximados.

\section{Atividades explorando simetrias no círculo trigonométrico}

Com estas atividades teve-se como objetivo usar as simetrias do círculo trigonométrico para relacionar seno, cosseno e tangente entre ângulos do $2^{\circ}, 3^{\circ}$ e $4^{\circ}$ quadrantes, comparando-os com os ângulos do primeiro quadrante, assim como reconhecer, via congruência de triângulos, as simetrias e, posteriormente, comparar seno, cosseno e tangente destes ângulos "simétricos". Para a execução desta proposta os estudantes construíram o círculo trigonométrico da Figura 11. 
Figura 11 - Círculo trigonométrico construído pelo estudante E6.

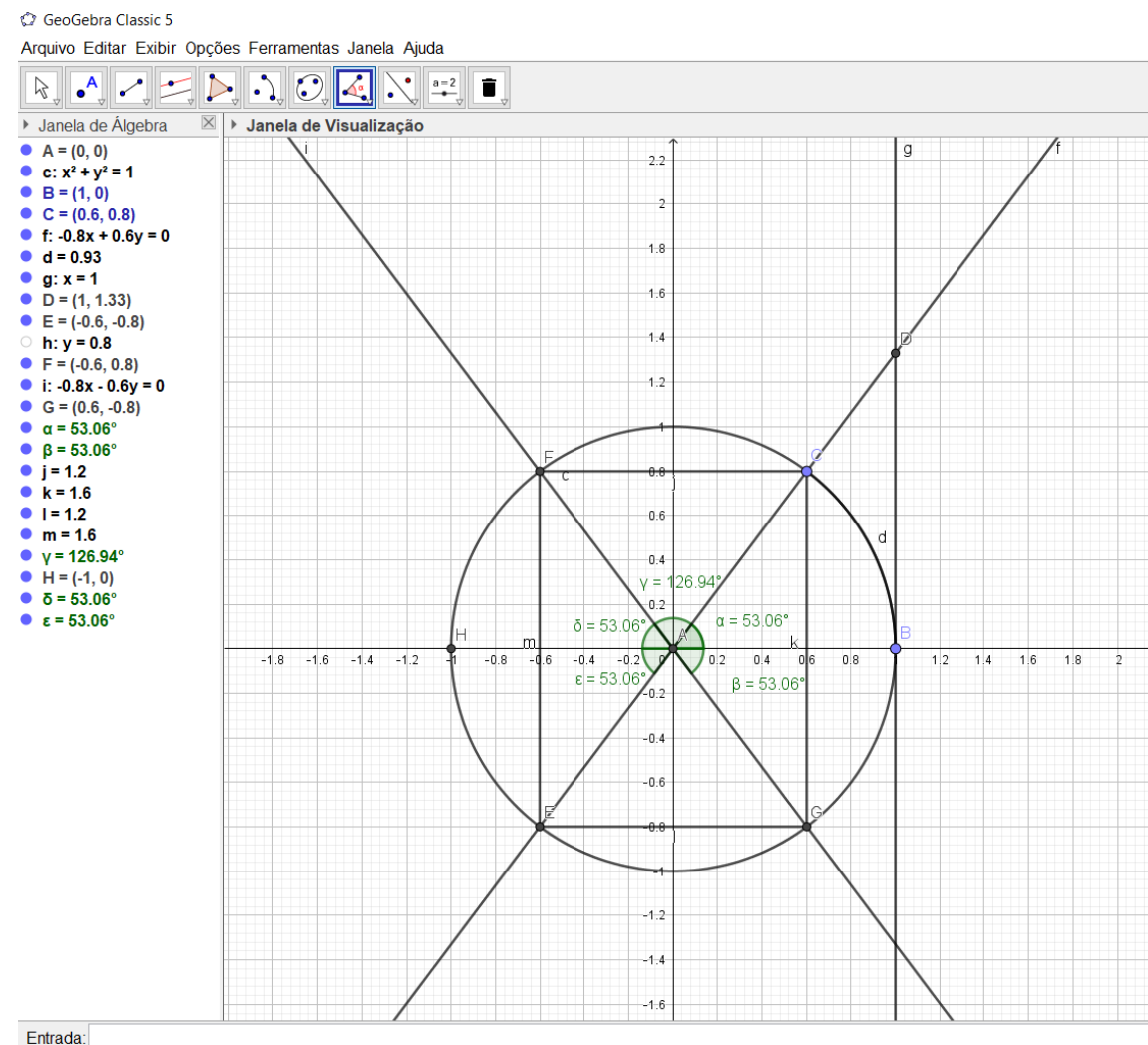

Fonte: Elaboração E6 (2019).

Essa atividade possibilitou estabelecer a simetria entre o primeiro quadrante com os demais quadrantes (segundo, terceiro e quarto) para o seno, cosseno e tangente em relação ao eixo vertical, horizontal e ao centro do círculo trigonométrico. Em seguida, os estudantes foram orientados a movimentar o ponto $\mathrm{C}$ na Figura 11, a fim de observar e comparar a variação do ângulo no primeiro com os demais quadrantes, bem como a sua simetria. Destacamos na Figura 12 a resolução do estudante E32. 
Figura 12 - Resolução do estudante E32

Atividade -4

1) Escrever um ângulo que tenha o mesmo valor de:
a) $\operatorname{sen}\left(30^{\circ}\right)=\operatorname{sen}\left(150^{\circ} \mathrm{C}\right.$
b) $\operatorname{sen}\left(45^{\circ}\right)=\operatorname{sen}\left(135^{\circ}\right)$
c) $\operatorname{sen}\left(60^{\circ}\right)=\operatorname{sen}\left(220^{\circ}\right)$
d) $\operatorname{sen}(x)=\operatorname{sen}\left(180^{\circ}-x\right.$
e) $\tan \left(30^{\circ}\right)=\tan (210) \mathrm{C}$
f) $\tan \left(45^{\circ}\right)=\tan \left(225^{\circ}\right)$
g) $\tan \left(60^{\circ}\right)=\tan (2406$
h) $\operatorname{tar}(x)=\tan \left(180^{\circ}+x \quad\right.$ sendo $x$ um ângulo do $1^{\circ}$ quadrante
i) $\cos \left(45^{\circ}\right)=\cos \left(365^{\circ} \mathrm{C}\right.$
k) $\cos \left(60^{\circ}\right)=\cos (370) \mathrm{C}$
1) $\cos (x)=\cos \left(360^{\circ}-x \mathrm{C}\right.$ sendo $x$ um ângulo do $1^{\circ}$ quadrante

2) Moviment
ou falso (F):
a) (V) $\operatorname{sen}(\pi-\alpha)=\operatorname{sen}(\alpha)$
b) (V) $\operatorname{sen}(\pi+\alpha)=-\operatorname{sen}(\alpha)$
c) (F) $\operatorname{sen}(2 \pi-\alpha)=\operatorname{sen}(\alpha) C$
d) (V) $\cos (\pi-\alpha)=-\cos (\alpha)$ c
e) $(F) \cos (\pi+\alpha)=\cos (\alpha)$
f) (V) $\cos (2 \pi-\alpha)=\cos (\alpha)$
g) (V) $\tan (\pi-\alpha)=-\tan (\alpha) \mathcal{L}$
h) $(\sqrt{ }) \tan (\pi+\alpha)=\tan (\alpha)$
i) (F) $\tan (2 \pi-\alpha)=\tan (\alpha) C$

3) Encontre os valores pedidos (usando se necessário, as identidades verdatici-as do exercicio anterior):
a) $\operatorname{sen}\left(120^{\circ}\right)=\operatorname{sen}\left(180^{\circ}-60^{\circ}\right)=\operatorname{sen}\left(60^{\circ}\right)=\frac{2}{2}$
b) $\operatorname{sen}\left(240^{\circ}\right)=\sin \left(180^{\circ}+60^{\circ}\right)=\operatorname{sen}\left(60^{\circ}\right)=-\frac{\sqrt{7}}{2}$
c) $\operatorname{sen}\left(315^{\circ}\right)=\sin \left(360^{\circ}+45^{\circ}\right)=\sin \left(45^{\circ}\right)=-\frac{\sqrt{2}}{2}$
d) $\cos \left(135^{\circ}\right)=\cos \left(180^{\circ}-45^{\circ}\right)-\cos \left(45^{\circ}\right)=-\frac{\sqrt{2}}{2}$
e) $\cos \left(210^{\circ}\right)=\cos \left(180^{\circ}+30^{\circ}\right)-\cos \left(30^{\circ}\right)=-\sqrt{3}$
f) $\cos \left(330^{\circ}\right)=\operatorname{Cor}\left(360^{\circ}-30^{\circ}\right)=6,\left(30^{\circ}\right)=\sqrt{3}$
g) $\tan \left(150^{\circ}\right)=\tan \left(180^{\circ}-30^{\circ}\right)=\tan \left(30^{\circ}\right)-\frac{\sqrt{3}}{3}$
h) $\tan \left(225^{\circ}\right)=\operatorname{Tan}\left(190^{\circ}+45^{\circ}\right)=\operatorname{Tan}\left(45^{\circ}\right)=13$
i) $\tan \left(300^{\circ}\right)=\operatorname{tar}\left(360^{\circ}-60^{\circ}\right)=\tan \left(60^{\circ}\right)=-\sqrt{3}$

Fonte: Elaboração E32 (2019).

Dos trinta e dois alunos presentes nessas aulas, vinte e cinco acertaram todos os itens propostos e sete erraram alguns dos itens. Gerar o círculo trigonométrico possibilitou que os discentes compreendessem a relação entre ângulos e seus correspondentes nos diversos 
quadrantes do círculo trigonométrico, bem como constatar os valores do seno, do cosseno e da tangente dos ângulos simétricos.

Com relação aos estudantes que erraram alguns dos itens, percebeu-se que tiveram dificuldade em relacionar o comprimento do arco com o ângulo no GeoGebra bem como no cálculo manual. Nos estudos de Gravina (2001), no que se refere ao uso da tecnologia como recurso de ensino, a autora relata que os ambientes de Geometria Dinâmica são ferramentas informáticas que permitem construção de objetos geométricos a partir das definições das propriedades. Em consonância com as pesquisas da autora, apresentou-se o material que possibilitou a geração do gráfico de cada função, relacionando o comprimento do arco, com o seno, cosseno e a tangente do ângulo.

Pela dinâmica da atividade, em decorrência da construção e da animação do gráfico, observaram-se o envolvimento e a participação dos estudantes. Os estudantes puderam perceber que, na construção dos gráficos, ambos poderiam ser construídos ao mesmo tempo, em diversas tonalidades de cores e com movimento, o que o tornou atrativo e diferente de trabalhar aulas de Matemática por meio do software.

Elaborar a sequência didática, acompanhar os alunos individualmente, foi imprescindível para a obtenção de um resultado satisfatório, ao encontro de Maia (2013), a utilização do software contribuiu para uma melhor compreensão dos conceitos matemáticos estudados. Em relação às vantagens da utilização do recurso tecnológico, esclarecem os autores:

\footnotetext{
O recurso tecnológico para ser utilizado deverá permitir explorar esses conceitos, dando oportunidades à sua compreensão por todos os alunos, desde os mais rápidos aos que apresentam maiores dificuldades. Por isso, deve possibilitar a experimentação, várias formas de resolução das questões (por ex. contar, operar, manipular, visualizar...) (AMADO; CARREIRA, 2015, p. 15).
}

Assim, nessa perspectiva, se o desenvolvimento dessa atividade ocorresse de forma tradicional, ou seja, utilizando a lousa e giz, demandaria muito tempo na construção de cada gráfico das funções, onde não possibilitaria que os estudantes visualizassem, interpretassem e analisassem os respectivos gráficos, além do fato de contar com a vantagem da agilidade e dinamismo. 


\section{Considerações finais}

As tecnologias estão cada vez mais presentes e praticamente em quase todas as atividades e vida da sociedade. Nesse contexto, torna-se um imperativo o professor estar atento às dinâmicas da sociedade e adequar as práticas de ensino consonantes e de acordo com essa realidade. Decorrente dessa necessidade, mesmo considerado um desafio pessoal do pesquisador, pelo fato que sempre estiveram presentes práticas pedagógicas tradicionais, como uso do quadro, giz e aulas expositivas, e também por sentir dificuldades em adotar metodologias de ensino mais dinâmicas nas práticas pedagógicas cotidianas, superaram-se essas dificuldades, resultando na proposta e no desenvolvimento da presente pesquisa.

Assim, a pesquisa teve como principal objetivo investigar como a utilização do software GeoGebra pode potencializar a exploração de tópicos da trigonometria. A partir do objetivo principal da pesquisa, decorreu o desenvolvimento dos específicos: o primeiro, foi verificar as potencialidades e promover a participação dos estudantes no estudo da trigonometria.

Perceberam-se uma interação e um envolvimento entre estudantes e professor, uma vez que, elaborar os gráficos das funções, garantiu a visualização de forma interativa e possibilitou que os estudantes criassem hipóteses, explorassem e propusessem alternativas, um trabalho coletivo que favoreceu a discussão e interatividade professor-estudante. A forma como esses recursos foram utilizados na pesquisa garantiu a efetividade do computador e do software GeoGebra como ferramentas pedagógicas, uma vez que foram potencializados e aproveitados na sala de aula.

Por meio da metodologia proposta na pesquisa, evidenciou-se que o uso do software GeoGebra pode ressignificar a forma de ensinar matemática, auxiliando na compreensão, no desenvolvimento e na explicação da disciplina, além da possibilidade de propiciar um ensino aos estudantes com capacidade para auxiliá-los na compreensão dos conteúdos a partir da utilização do simbolismo matemático envolvido, a fim de oportunizar o raciocínio e a autonomia dos estudantes na realização de tarefas e desenvolver a capacidade de resolver exercícios e problemas contextualizados, por meio do uso do computador.

Por fim, apontou ainda que a forma de ensinar matemática pode ser reinventada por meio do uso de softwares computacionais, mediante uma prática pedagógica diferenciada utilizada junto aos estudantes para que haja a compreensão da matemática enquanto uma disciplina que perpassa as diferentes dimensões da vida humana. O uso do software na 
perspectiva do desenvolvimento de uma metodologia ativa, dinâmica e inovadora viabiliza o elo e a interpretação do compromisso social na produção do conhecimento.

\section{Referências}

AMADO, N. M. P.; CARREIRA, S. P. Recursos Tecnológicos no Ensino e Aprendizagem da Matemática. In: DULLIUS, Maria Madalena; QUARTIERI, Marli Teresinha (Orgs.). Explorando a Matemática com Aplicativos Computacionais - Anos Iniciais do Ensino Fundamental. Lajeado: Ed. da Univates, 2015.

BORBA, M. C. Informática e Educação Matemática. 5. ed. Belo Horizonte: Autêntica, 2016.

BRASIL. Ministério da Educação. Plano Nacional de Educação (2014-2014). Lei n. ${ }^{\circ} 13.005$ de 25 de junho de 2014.

BRASIL. Ministério da Educação. Base Nacional Comum Curricular. Portaria $\mathrm{n}^{\mathrm{o}}$ 1.570, publicada no D.O.U. de 21/12/2017, secção 1, p. 146. Disponível em:

http://basenacionalcomum.mec.gov.br/images/historico/BNCC_EnsinoMedio_embaixa_site_ 110518.pdf. Acesso em: 10 jan. 2019.

DULliUS, M. M.; HAETINGER, C. Ensino e Aprendizagem de Matemática em Ambientes Informatizados: Concepção, Desenvolvimento, Uso e Integração Destes no Sistema Educacional. Anais IV Encontro Ibero-Americano de Coletivos Escolares e Redes de Professores que fazem investigação na sua escola. Porto Alegre, 2004. Disponível em: http://www.if.ufrgs.br/ienci/uploaded/ATA_EIBIEC_IV.pdf. Acesso em: 05 jun. 2019.

DULLIUS, M. M.; QUARTIERI, M. T. Maria Madalena Dullius, Marli Teresinha Quartieri (Org.). Explorando a matemática com aplicativos computacionais: anos iniciais do ensino fundamental. Lajeado: Editora Univates, 2015. p. 5-6. Disponível em:

https://www.univates.br/editora-univates/media/publicacoes/144/pdf_144.pdf. Acesso: 7 fev. 2018.

GIL, A. C. Métodos e Técnicas de Pesquisas Sociais. São Paulo: Atlas, 2008. v.6.

GRAVINA, M. A. Os ambientes de geometria dinâmica e o pensamento hipotético dedutivo. 2001. 277f. Tese (Doutorado em Informática na Educação). Universidade Federal do Rio Grande do Sul. Porto Alegre, 2001. Disponível em:

https://www.lume.ufrgs.br/bitstream/handle/10183/2545/000321616.pdf?sequen. Acesso em: 05 jun. 2019.

GRAVINA, M. A. et al. (org.). Matemática, Mídias Digitais e Didática: tripé para formação do professor de Matemática. Porto Alegre: Evangraf, 2012. p. 37-52.

LIMA, E. L.; CARVALHO, P. C. P.; MORGADO, A. C. A matemática do ensino médio: Volume 1, 8. ed. Rio de Janeiro: SBM, 2005. 
MAIA, J. O ensino de funções trigonométricas através do software GeoGebra. 2013. 48f. Dissertação (Mestrado em Matemática). Programa de Pós-Graduação em Matemática em Rede Nacional, Universidade Federal do Rio Grande do Norte, 2013.

NASCIMENTO, C. C. P. O uso do GeoGebra no ensino das funções trigonométricas no $\mathbf{2}^{\circ}$ ano do Ensino Médio no IFMT Campus Cuiabá. 2019. 130f. Dissertação (mestrado) Programa de Pós-Graduação em Ensino de Ciências Exatas, Lajeado, 2019a.

NASCIMENTO, C. C. P. Sequência didática para o ensino das funções trigonométricas com o uso do software GeoGebra. Universidade do Vale do Taquari - Univates. Programa de Pós-Graduação em Ensino de Ciências Exatas, Lajeado, 2019b. Acesso em: 27 dez. 2019. Disponível:

https://www.univates.br/ppgece/media/pdf/2019/carlos_carlao_pereira_do_nascimento.pdf

NEIDE, I. G.; QUARTIERI, M. T. Recursos Tecnológicos nos processos de ensino e de aprendizagem da matemática e da física. In: DULLIUS, Maria Madalena; QUARTIERI, Marli Teresinha (Org.). Aproximando a Matemática e a Física por meio de recursos tecnológicos: Ensino Médio. Lajeado: Ed. Da Univates, 2016. p. 9-14. Disponível em: https://www.univates.br/editora- univates/media/publicacoes/191/pdf_191.pdf. Acesso: 20 maio 2019.

PAIVA, M. H. P. Aprendizagem de Frações com Softwares e aplicativos matemáticos online. 2016. 112f. Dissertação (mestrado) - Programa de Pós-Graduação Stricto Sensu Mestrado Profissional em Ensino de Ciências Exatas do Centro Universitário UNIVATES. Lajeado, 2016.

SCALDELAI, D. O software GeoGebra. In: BASNIAK, Maria Ivete; ESTEVAM, Everton José Golgoni (Orgs.). O GeoGebra e a Matemática da Educação Básica: frações, estatística, círculo e circunferência. Curitiba: Ithala, 2014. p. 13-23.

VALENTE, J. A. V. O uso inteligente do computador na educação. Pátio Revista Pedagógica, Porto Alegre, Ano I, n. 1, p. 19-21, mai/jul, 1997. 